\title{
SlipBuddy: A Mobile Health Intervention to Prevent Overeating
}

\author{
Bengisu Tulu \\ Worcester Polytechnic Institute \\ bengisu@wpi.edu \\ Joseph Acheson \\ Worcester Polytechnic Institute \\ jracheson@wpi.edu \\ Gage Heeringa \\ Michigan Technological \\ University \\ grheerin@mtu.edu
}

\author{
Carolina Ruiz \\ Worcester Polytechnic Institute \\ ruiz@cs.wpi.edu \\ Andrew Busch \\ Worcester Polytechnic Institute \\ abbusch@wpi.edu \\ Victor Jaskula \\ Skidmore College \\ vjaskula@skidmore.edu
}

Sherry Pagoto

University of Massachusetts Medical School sherry.pagoto@umassmed.edu

\author{
Joshua Allard \\ Worcester Polytechnic Institute \\ jnallard@wpi.edu \\ Andrew Roskuski \\ Worcester Polytechnic Institute \\ ajroskuski@wpi.edu \\ Jessica Oleski \\ University of Massachusetts \\ Medical School \\ jessica.oleski@umassmed.edu
}

\begin{abstract}
Obesity is one of the top health issues around the globe. Rapid adoption of smartphones presents an opportunity for delivering technology-based interventions that are designed to tackle behaviors that contribute to weight gain. Research shows that the vast majority of weight loss apps in the market place do not go beyond deploying tracking based strategies that are burdensome to the users. In this study, we present a new mobile app and an intervention system called SlipBuddy that puts less burden on users and implements stimulus control strategy to help users lose weight. We describe the SlipBuddy system in detail and present the results of the first phase of a pilot study. Our findings indicate that a mobile app that simply helps users identify and track overeating episodes can potentially result in weight loss.
\end{abstract}

\section{Introduction}

Obesity affects $13 \%$ of the world's population, a rate that has doubled since 1980 [1]. Rates of obesity in the US are even worse, with $1 / 3$ of the population affected [2]. Lifestyle interventions involve nutrition, exercise, and behavioral modification counseling and have been shown to be effective in reducing weight and preventing chronic disease [3]. Unfortunately, such interventions are expensive and require numerous patient visits with highly trained professionals over 6-12 months [4].

The use of smartphones has skyrocketed globally in the past decade, which has provided new ways to communicate with and educate patients. Mobile technology has the capacity to deliver evidence-based weight loss interventions with lower cost and burden than traditional intervention models. However, studies report that their potential is yet to be realized [5]. A recent literature review of mobile technology interventions illustrates the limited use of mobile technology, mainly text messaging, in such interventions [6]. Although many commercial mobile apps for weight management are available in the marketplace, they use a very narrow range of evidence-based strategies [7]. Moreover, studies show that they do not produce weight loss without more intensive treatment [8].

One drawback of commercial apps is that the key strategy employed is dietary and physical activity self-monitoring, a tedious process that requires the user to enter every food they have consumed and all exercise performed each day. While some users persist with this activity for weeks or months, many have difficulty initiating this task or persisting for more than a month [8]. Studies report that the apps in this space need to go beyond tracking, and deliver more personalized interventions through the integration of evidence-based theories and advanced technologies such as machine learning $[7,9,10]$.

Mobile apps that implement evidence-based behavioral weight loss strategies with less burden 
could improve the impact of technology on the obesity epidemic. We explored how stimulus control, a common behavioral weight loss strategy, can be implemented in a mobile app and an intervention management system designed to deliver personalized interventions. In this paper, we describe the SlipBuddy system and present results from the initial phase of an mHealth intervention that utilizes SlipBuddy to deliver personalized messages to help users identify and avoid overeating stimuli.

\section{Background}

Dietary self-monitoring is a key behavioral strategy that is strongly associated with weight loss success in studies [11], but it is not clear how thorough a user has to be. Ample research shows that most individuals underreport when self-monitoring. One approach to reducing the intensity of dietary self-monitoring is to have individuals only record overeating episodes, meaning episodes of eating or drinking in which they consumed more than intended or at a time they did not intend to eat. This focuses the patient on the segment of their eating behavior that should be modified, rather than having them record all eating episodes, many of which may be portion-controlled, involve healthy choices, and require no modification. It may not be necessary for the user to spend time monitoring healthy choices, but instead spend time focusing exclusively on the unhealthy choices.

Stimulus control is a strategy in which the patient learns to 1) identify and avoid stimuli, or contextual factors, that trigger an unhealthy behavior, and 2) insert new stimuli into the environment that trigger healthy behaviors, ideally behaviors that are incompatible with the unhealthy behavior [12]. For example, if a patient overeats in front of the TV, the TV is likely an overeating trigger. Avoiding the TV or engaging in activities that are incompatible with eating while watching TV (e.g., walking on the treadmill) can help break the connection between TV and eating.

\section{SlipBuddy}

We designed and developed a technology intervention solution, illustrated in Figure 1, composed of a mobile app client called SlipBuddy, a server and a web based provisioning system for clinicians to monitor users' progress and to manage delivery of individually tailored interventions. The following sections describe each component in detail.

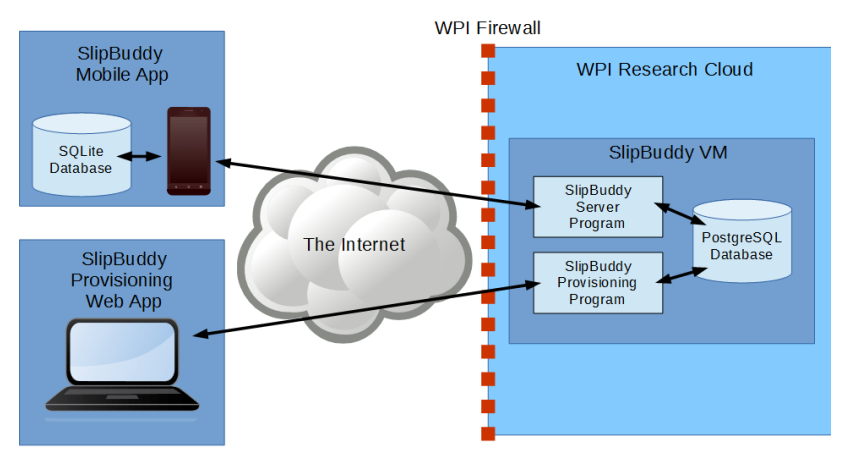

Figure 1. SlipBuddy intervention solution

\subsection{SlipBuddy mobile app}

SlipBuddy, an Android app, is developed to gather data related to users' overeating episodes and to deliver behavioral modification interventions to help them focus on reducing the frequency of overeating episodes as a way to decrease overall intake. In addition to streamlining the process of selfmonitoring, SlipBuddy also employs the evidencebased behavioral strategy referred to as stimulus control.

SlipBuddy users hit an "Oops!" button each time they overeat (see Figure 2). In order to leverage stimulus control, the app collects user-entered and passively sensed data after the user presses the Oops! button. Data collected include timestamp for data entry, episode date and time, levels of stress and hunger, activity being performed before or during the episode (e.g., working, socializing, watching TV, commuting), location, and type of meal associated with overeating (e.g., lunch or a snack). Users who need help determining if an eating episode is overeating can press "What is a Slip?" button to see a description of when they should press the button. The message displayed says "Report any eating that you feel resulted in your consuming (food or drink) more than planned at a meal or between meals. Examples might include having a donut at the office after you already had breakfast, eating an extra helping at dinner, grabbing a candy bar from the vending machine instead of the apple you brought for a snack, having a snack at night when you intended to stop eating for the night, having too many alcoholic drinks, etc."

SlipBuddy also collects contextual data regularly during a day. Three check-ins are built into the app. The morning check-in, scheduled for 9:00 AM, asks five questions to collect data about morning stress and hunger levels, total number of hours slept and level of restedness, and daily weigh-in value. The afternoon check-in, scheduled for 2:00 PM, asks only about stress levels. The evening check-in, scheduled 
for 8:00 PM, asks about stress level at the time and provides the opportunity to record any overeating episodes that were not reported during the day. SlipBuddy allows users to complete check-ins for the current and the previous days.

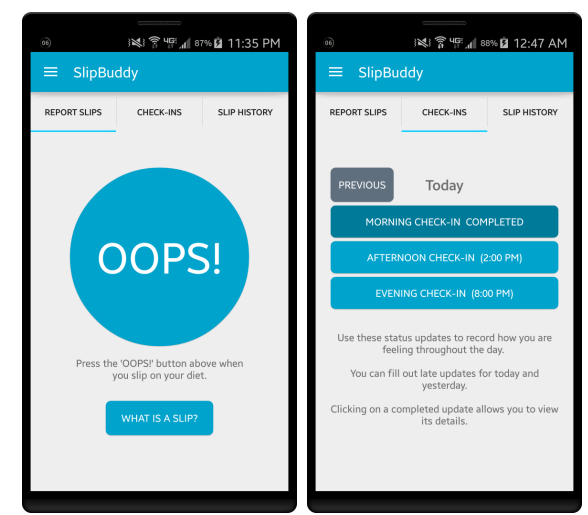

Figure 2. SlipBuddy main interface and check-ins tab

After a period of use, each user's SlipBuddy data is analyzed to generate a predictive model that directs the app to display a message to the user when they are in a situation that in the past has triggered overeating with a suggestion for how to manage that particular trigger (see Figure 3). For example, if a user often overeats when stressed, the app would nudge the user with a suggestion that says, "Your stress level is high! According to your data, you overeat more when stressed. Try reducing stress with an activity that doesn't involve eating."
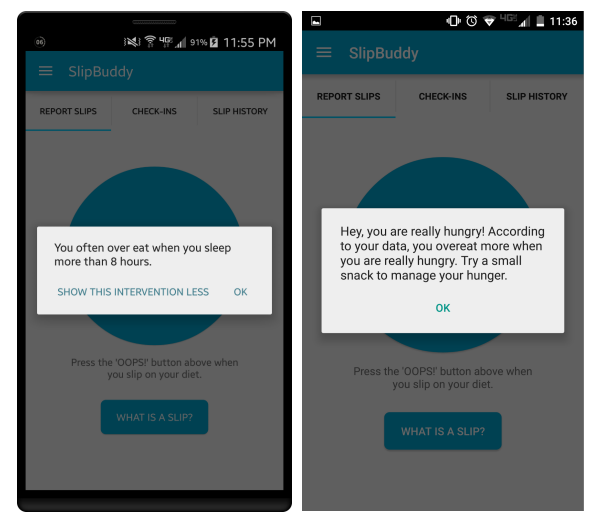

Figure 3. SlipBudy intervention dialogs

Figure 4 provides an overview of the SlipBuddy architectural design. SlipBuddy Android app starts with the Main Activity, the first activity loaded when the app is opened. There are three components to the Main Activity: the Tab Controller, the Navigation Drawer, and the Notification Manager.

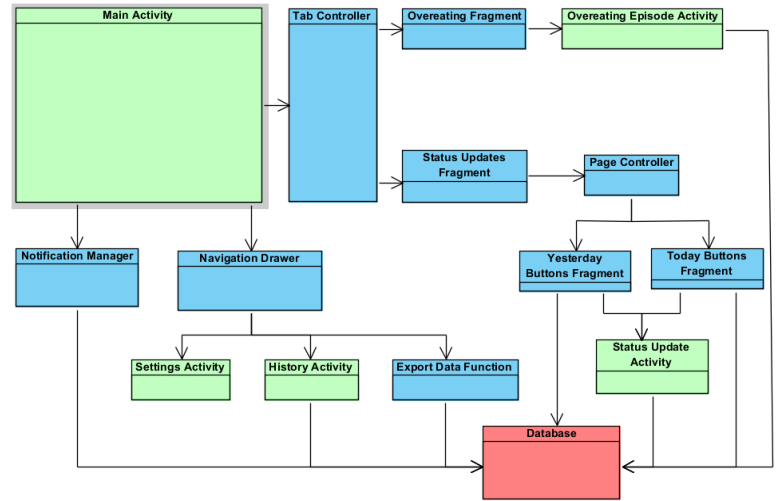

Figure 4. SlipBuddy interaction layout

The Tab Controller divides the two main data entry portions of the app and controls the tabbed view of the Main Activity. The left tab, the Overeating Fragment, opens the Overeating Episode Activity for entering overeating episodes to be stored in the Database. The right tab, the Status Updates Fragment, controls the Check-in functions and has an instance of the Page Controller that allows for swapping between its fragments (Yesterday Buttons Fragment and Today Buttons Fragment) using next and previous buttons. Upon selecting any of the incomplete status update buttons within these fragments, the Status Update Activity opens to allow the user to create a status update. These status updates are stored by the Activity into the Database. If the selected Check-in has been completed, the Activity will access the Database and display the completed status update values.

The Navigation Drawer shows the settings for the application and contains the Settings Activity, the History Activity, and the Export Data Function. The Settings Activity contains the settings that can be altered by the user. The settings are defined in an xml file. The History Activity displays a scroll of all overeating episodes that have previously been entered. The Export Data Function exports the database object to a local directory. An SQLite database is used on the mobile device to store data locally. The Notification Manager handles the notifications that the app displays. It accesses the Database to load stored settings.

\subsection{SlipBuddy server}

The server is designed and developed to facilitate scalable implementation of mobile health interventions. The data entered by users to SlipBuddy is regularly sent to the provisioning server using a sync service in the app using JSON format. The sync process is triggered every time new data is entered in 
the client application, and will retry after a waiting period if the process fails. A sync metadata SQLite database is set up on the client, in the same configuration as the main client database, so that only new data will be sent to the server.

The server side is implemented using an HTTPS server connected to a PostgreSQL database. The server software is written in Java, and receives client requests and commits the data to the database. The requests are sent encrypted using the user's password as a key. The server also has the ability to verify that a user's password is correct by attempting to decrypt a phrase known to both the client and the server, which has been encrypted using the password provided by the client. Once interventions were implemented, they were sent to the clients in the response message for a successful sync operation.

\subsection{SlipBuddy provisioning application}

The SlipBuddy provisioning web application is developed to help manage and monitor interventions remotely. The web application, developed using Node.js and Jade templates, connects to SlipBuddy server's PostgreSQL database in order to retrieve and interact with the data. To protect the privacy of user data, we have implemented several security features, such as using HTTPS with certified credentials and assigning users secure server-generated passwords.

The provisioning interface provides authenticated administrative-users (e.g., healthcare professionals responsible for running the intervention program) the ability to view SlipBuddy user IDs and their associated passwords, overeating episodes, check-ins, and recorded locations. Through the provisioning interface, authenticated administrative-users can create new users with auto-generated passwords and enable a SlipBuddy user to start receiving interventions. The ability to create new interventions on the 'Interventions' page, illustrated in Figure 5, is also provided to select administrative-users.

\section{Methodology}

We started conducting a pilot field study in November 2015 to evaluate SlipBuddy intervention. This pilot study consisted of two phases: (1) Assessment Phase and (2) Feedback Phase. Although we will describe procedures for both phases in this paper, we will only report results from the Assessment Phase since the Feedback Phase is still ongoing for some participants.

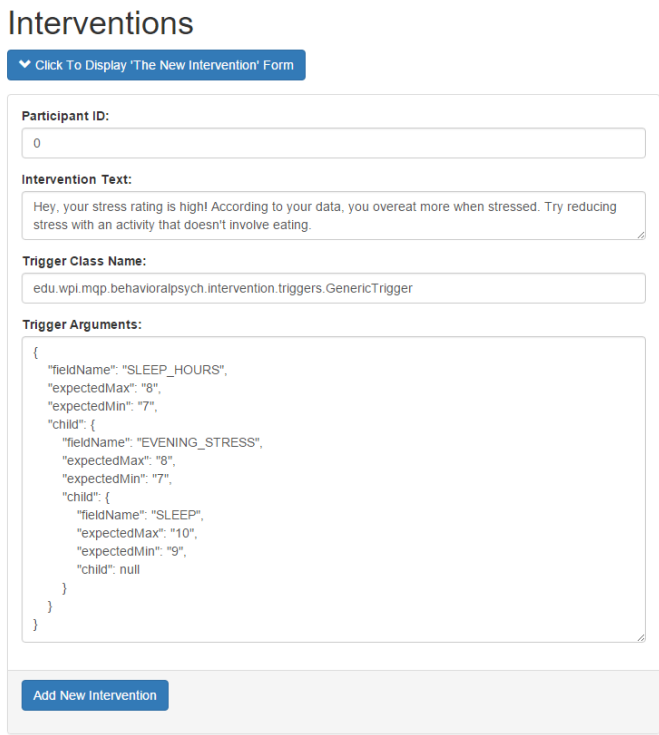

Figure 5. Intervention interface

\subsection{Participant recruitment}

Participants were required to be over the age of 18 and have an Android smartphone running version 4.0 or later to install and use the SlipBuddy app. In addition, participants were either overweight $(25.0 \leq$ $\mathrm{BMI}<30)$ or obese $(\mathrm{BMI} \geq 30)$, were familiar with using mobile applications, and had an interest in losing weight. Participants were excluded if they were not willing to record their stress and weight on the app, did not use their phone daily, were unable to provide consent, were pregnant, or were prisoners.

Participants were recruited in two waves. The first wave consisted of participants recruited from the local community using flyers, electronic ads, and newsletters. Interested participants called the study staff to learn more about the study. During this call, study staff provided a detailed overview of the study procedures and asked questions related to eligibility. Eligible participants attended an in-person 1-hour baseline appointment. This appointment included informed consent, assessments, orientation to the study procedures, and mobile app installation. During this visit, participants downloaded the SlipBuddy mobile application to their phones and received instructions on how to use the app during the first phase of the study. Participants were telephoned one week after starting the app to make sure it was working properly, to answer any questions, and address technical difficulties.

Participants in the second wave were recruited at a national level through social media and web-based advertisement. All study procedures were performed online or over the phone. All procedures were 
identical as the first wave, except the online participants completed an initial pre-screening survey and communicated with staff for the baseline appointment via phone instead of in person. The online recruitment was chosen in an effort to complete the pilot in a short period of time and to maximize generalizability by recruiting across the country.

\subsection{Study procedures}

The first month of app use was considered the Assessment Phase. Participants were asked to provide status updates using the app three times a day by responding to a set of questions, and to record any overeating episodes that occurred during the day, as described in Section 3.1. When they overate, they were instructed to open the app and click on the Oops! button and answer the questions related to their overeating episode. At the end of the one-month Assessment Phase, participants completed an individual interview to discuss their experience using the app, likes and dislikes, the type of feedback they would like to receive during the Feedback Phase, how they would like feedback messages to look, and how they wished to receive them. After the interview, participants took a two-month break from using the app while the study team analyzed both the collective and individual user data, and developed customized interventions based on the assessment data.

During the Feedback Phase, participants resumed use of the app for one month as they did in the Assessment Phase; they also received interventions that popped up when a participant was at risk of having an overeating episode based on past behavior. Feedback messages told participants that they were highly likely to overeat given their specific current circumstances (e.g., slept less than 6 hours and morning stress was high). An end-of-study interview was conducted at the end of the Feedback Phase to discuss participants' overall opinions of the mobile application and feedback reports. The interview included questions related to adherence to use of the app, acceptability of the mobile application, technical difficulties, and reactions to the feedback messages.

\subsection{Data analysis}

Prior to data analysis, participants' demographic data and app data were merged into a single data set. App data included check-ins and overeating episodes reported by participants using the app during the Assessment Phase of the pilot study.

Univariate analysis was conducted using MS Excel. Frequencies and percentages were calculated based on several demographic variables to understand how the data was distributed across different groups. Next we conducted bivariate analysis by constructing a Pearson correlation matrix using IBM SPSS software to understand the relationships between various factors including demographic variables and variables collected by the app. We then performed multivariate analysis to determine associations among groups of variables.

The bivariate and multivariate analyses helped us identify collections of variables that best predicted overeating behavior. Using these variables we constructed predictive models using decision trees. The decision trees machine learning technique is a powerful and widely used approach to discover predictive patterns in data and to conveniently represent these patterns in a compact tree structure. In order to construct decision trees, we used the Weka system's J48 implementation of the popular C4.5 decision tree algorithm [13]. In generating these trees, $k$-fold cross-validation $(k=10)$ was used to avoid overfitting. Additionally, we used Python's Pandas, NumPy, and Matplotlib libraries to visualize our data.

\section{Results}

This section reports the results of our analysis performed over the data collected from participants during the Assessment Phase.

\subsection{Data sample}

Sixteen participants, who satisfied the requirements described in Section 4.1, were recruited for the pilot study. Each of these participants followed the study protocol described in Section 4.2.

Table 1 summarizes relevant characteristics of the set of participants. Participants' gender, age, education level, household annual income, and initial weight varied widely, providing a good sample for the pilot study. Participant adherence to the app was notably high, with a small number of missed checkins as Figure 6 shows. Participants reported overeating episodes on a daily basis, as Figure 7 illustrates.

Using the check-ins and overeating episodes reported by the participants during the Assessment Phase together with demographic information collected at the beginning of the pilot study, we assembled a dataset for analysis. This dataset consists of 352 data entries, each one corresponding to a single day of app data recorded per participant (22 days $\times 16$ participants $=352$ data entries). While most participants used SlipBuddy for more than 22 
days, with a few participants continuing to use it even after the end of the Assessment Phase, one participant had only 22 daily records. To give equal representation of all participants, the data was fixed to contain information of days 1 through 22 for each participant.

Each of the 352 data entries consists of the participant's demographic information, the responses provided to the questions asked during 3 daily checkins and the number of overeating episodes reported for that day. Other derived variables (e.g., weight change during the study and number of overeating episodes recorded in previous day) were added to the dataset. Table 1 and Table 2 present some of the variables in the dataset.

Table 1. Summary participant data during the 1-month Assessment Phase (SD: Standard Deviation)

\begin{tabular}{|c|c|c|c|c|}
\hline & Number (\%) & $\begin{array}{l}\text { Body } \\
\text { in Ibs } \\
\text { Mean } \pm \mathrm{SD}\end{array}$ & $\begin{array}{l}\text { ight Change } \\
\text { as } \% \text { of initial weight } \\
\text { Mean } \pm \text { SD }\end{array}$ & $\begin{array}{c}\text { Total Number of } \\
\text { Overeating Episodes } \\
\text { Mean } \pm \text { SD }\end{array}$ \\
\hline $\begin{array}{l}\text { Gender } \\
\text { female } \\
\text { male }\end{array}$ & $\begin{array}{r}10(62.5 \%) \\
6(37.5 \%) \\
\end{array}$ & $\begin{array}{l}-0.60 \mathrm{lbs} \pm 2.73 \\
-5.00 \mathrm{lbs} \pm 4.04\end{array}$ & $\begin{array}{l}-0.42 \% \pm 1.40 \\
-2.10 \% \pm 1.90 \\
\end{array}$ & $\begin{array}{l}12.30 \pm 8.94 \\
16.33 \pm 10.06\end{array}$ \\
\hline $\begin{array}{l}\text { Age } \\
20-39 \\
40-49 \\
50-59 \\
60-69 \\
\end{array}$ & $\begin{array}{l}6(37.5 \%) \\
4(25 \%) \\
4(25 \%) \\
2(12.5 \%) \\
\end{array}$ & $\begin{array}{l}-0.83 \mathrm{lbs} \pm 3.34 \\
-3.75 \mathrm{lbs} \pm 4.32 \\
-3.25 \mathrm{lbs} \pm 4.02 \\
-1.50 \mathrm{lbs} \pm 2.50 \\
\end{array}$ & $\begin{array}{l}-0.37 \% \pm 1.51 \\
-1.94 \% \pm 1.99 \\
-1.33 \% \pm 1.75 \\
-0.72 \% \pm 1.36 \\
\end{array}$ & $\begin{array}{l}15.50 \pm 11.27 \\
12.75 \pm 9.93 \\
10.50 \pm 5.17 \\
17.50 \pm 7.50 \\
\end{array}$ \\
\hline $\begin{array}{l}\text { Marital Status } \\
\text { Single } \\
\text { Married } \\
\text { Divorced / Widowed / Other }\end{array}$ & $\begin{array}{c}3(18.75 \%) \\
10(62.5 \%) \\
3(18.75 \%) \\
\end{array}$ & $\begin{array}{r}2.00 \mathrm{lbs} \pm 2.16 \\
-2.50 \mathrm{lbs} \pm 3.01 \\
-5.67 \mathrm{lbs} \pm 4.11 \\
\end{array}$ & $\begin{array}{r}0.86 \% \pm 0.85 \\
-1.09 \% \pm 1.38 \\
-2.80 \% \pm 1.86 \\
\end{array}$ & $\begin{aligned} & 8.33 \pm 6.94 \\
& 16.10 \pm 10.73 \\
& 11.67 \pm 2.36 \\
&\end{aligned}$ \\
\hline $\begin{array}{l}\text { Education } \\
\text { High school /some college } \\
\text { Trade school / Assoc.'s degree } \\
\text { Bachelor's degree } \\
\text { Some grad school / Master's } \\
\text { degree }\end{array}$ & $\begin{array}{l}4(25 \%) \\
3(18.75 \%) \\
6(37.5 \%) \\
3(18.75 \%)\end{array}$ & $\begin{array}{l}-3.00 \mathrm{lbs} \pm 3.94 \\
-5.67 \mathrm{lbs} \pm 4.11 \\
-1.00 \mathrm{lbs} \pm 3.42 \\
-0.33 \mathrm{lbs} \pm 1.25\end{array}$ & $\begin{array}{l}-1.30 \% \pm 1.74 \\
-2.62 \% \pm 1.89 \\
-0.61 \% \pm 1.65 \\
-0.01 \% \pm 0.48\end{array}$ & $\begin{array}{l}12.50 \pm 8.26 \\
12.33 \pm 3.30 \\
17.33 \pm 12.71 \\
10.00 \pm 4.55\end{array}$ \\
\hline $\begin{array}{l}\text { Ethnicity } \\
\text { Caucasian; non-Hispanic White } \\
\text { Other }\end{array}$ & $\begin{array}{r}13(81.25 \%) \\
3(18.75 \%) \\
\end{array}$ & $\begin{array}{c}-3.08 \mathrm{lbs} \pm 3.65 \\
1.33 \mathrm{lbs} \pm 2.87 \\
\end{array}$ & $\begin{array}{c}-1.44 \% \pm 1.70 \\
0.66 \% \pm 1.08 \\
\end{array}$ & $\begin{array}{l}14.00 \pm 10.26 \\
13.00 \pm 5.72 \\
\end{array}$ \\
\hline $\begin{array}{l}\text { Work Status } \\
\text { Employed full-time } \\
\text { Employed part-time } \\
\text { Disabled } \\
\text { Other } \\
\end{array}$ & $\begin{array}{l}8(50 \%) \\
3(18.75 \%) \\
3(18.75 \%) \\
2(12.5 \%) \\
\end{array}$ & $\begin{array}{l}-0.88 \mathrm{lbs} \pm 2.85 \\
-2.00 \mathrm{lbs} \pm 2.16 \\
-5.00 \mathrm{lbs} \pm 4.90 \\
-4.00 \mathrm{lbs} \pm 5.00 \\
\end{array}$ & $\begin{array}{l}-0.42 \% \pm 1.28 \\
-1.18 \% \pm 1.28 \\
-2.23 \% \pm 2.35 \\
-1.59 \% \pm 2.16\end{array}$ & $\begin{aligned} 13.63 & \pm 10.48 \\
19.00 & \pm 11.43 \\
12.33 & \pm 3.30 \\
9.00 & \pm 4.00\end{aligned}$ \\
\hline $\begin{array}{l}\text { Num. persons living in } \\
\text { household } \\
1 \\
2 \\
3-4 \\
5-8\end{array}$ & $\begin{array}{l}3(18.75 \%) \\
7(43.75 \%) \\
3(18.75 \%) \\
3(18.75 \%) \\
\end{array}$ & $\begin{array}{l}-3.67 \mathrm{lbs} \pm 6.60 \\
-2.57 \mathrm{lbs} \pm 3.20 \\
-1.00 \mathrm{lbs} \pm 1.63 \\
-1.33 \mathrm{lbs} \pm 2.62 \\
\end{array}$ & $\begin{array}{l}-1.94 \% \pm 2.97 \\
-1.05 \% \pm 1.39 \\
-0.67 \% \pm 1.12 \\
-0.52 \% \pm 1.22\end{array}$ & $\begin{array}{l}14.33 \pm 3.30 \\
14.29 \pm 12.09 \\
14.00 \pm 10.98 \\
12.00 \pm 3.56\end{array}$ \\
\hline $\begin{array}{l}\text { Avg. estimated household } \\
\text { annual income } \\
\$ 0-\$ 25000 \\
\$ 35,001-\$ 40,000 \\
\$ 45,001-\$ 60,000 \\
\text { Over } \$ 75,001 \\
\end{array}$ & $\begin{array}{l}3(18.75 \%) \\
2(12.5 \%) \\
4(25 \%) \\
7(43.75 \%)\end{array}$ & $\begin{array}{c}-5.00 \mathrm{lbs} \pm 4.90 \\
2.50 \mathrm{lbs} \pm 2.50 \\
-4.25 \mathrm{lbs} \pm 3.56 \\
-1.29 \mathrm{lbs} \pm 1.67\end{array}$ & $\begin{array}{c}-2.23 \% \pm 2.35 \\
1.01 \% \pm 1.01 \\
-1.98 \% \pm 1.63 \\
-0.60 \% \pm 0.85\end{array}$ & $\begin{array}{l}10.00 \pm 5.72 \\
10.00 \pm 8.00 \\
15.75 \pm 5.63 \\
15.43 \pm 12.01\end{array}$ \\
\hline $\begin{array}{l}\text { Initial body weight (lbs) } \\
140-175 \\
176-200 \\
201-250 \\
251-330 \\
\end{array}$ & $\begin{array}{l}4(25 \%) \\
4(25 \%) \\
4(25 \%) \\
4(25 \%) \\
\end{array}$ & $\begin{array}{l}-0.25 \mathrm{lbs} \pm 1.64 \\
-2.25 \mathrm{lbs} \pm 2.38 \\
-5.00 \mathrm{lbs} \pm 6.16 \\
-1.50 \mathrm{lbs} \pm 1.66 \\
\end{array}$ & $\begin{array}{l}-0.21 \% \pm 1.12 \\
-1.20 \% \pm 1.29 \\
-2.26 \% \pm 2.68 \\
-0.52 \% \pm 0.60\end{array}$ & $\begin{array}{l}12.75 \pm 9.76 \\
13.75 \pm 7.40 \\
14.50 \pm 3.20 \\
14.25 \pm 14.32 \\
\end{array}$ \\
\hline Total & $16(100 \%)$ & $-2.25 \mathrm{lbs} \pm 3.91$ & $-1.05 \% \pm 1.80$ & $13.81 \pm 9.58$ \\
\hline
\end{tabular}




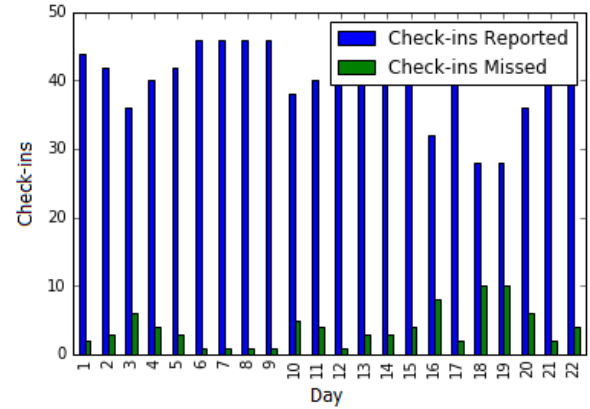

Figure 6. Daily check-ins during the Assessment Phase

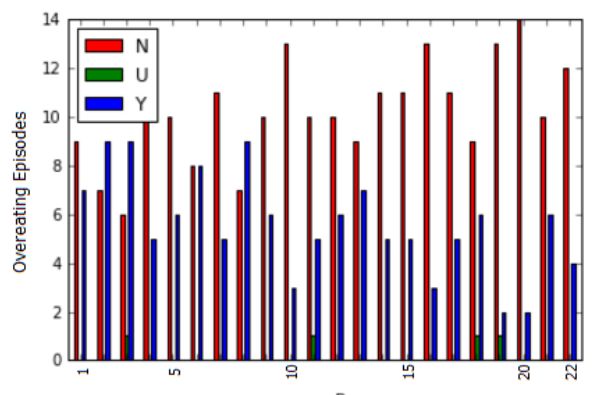

Day

Figure 7. Daily reporting of overeating ( $\mathrm{N}$ : no episode reported; Y: episode reported; U: unknown due to no check-in activity)

\subsection{Group-based data analysis and predictive modeling}

According to the univariate and bivariate analyses presented in Table 1, males lost more weight on average than females even though males reported a higher number of overeating episodes on average. Middle-aged participants (40-59 years) reported fewer overeating episodes and more weight loss on average compared to younger (20-39) and older (6069) participants. Married participants exhibited a higher number of overeating episodes whereas participants who live alone experienced a higher weight loss than participants who live with others. Furthermore, part-time employees recorded the highest number of overeating episodes in average $(19.00 \pm 11.43)$ while participants who are single recorded the lowest number in average $(8.33 \pm 6.94)$. Out of the 16 participants, 9 lost weight (with an average of $-4.9 \mathrm{lbs} \pm 3.03$; or $-2.3 \% \pm 1.37$ ), $3 \mathrm{kept}$ the same weight, and 4 gained some weight (with an average of $2 \mathrm{lbs} \pm 1.73$; or $0.97 \% \pm 0.61$ ) while using the app during the one-month Assessment Phase.

Pairwise Pearson correlations (calculated using SPSS and shown in Table 2) provide additional insights. There are statistically significant positive pairwise correlations among education level, morning stress, and morning hunger (note that the hunger scale goes from 0 , extremely hungry, to 10 , uncomfortably full). Similarly for education level, and morning, afternoon, and evening stress. Both gender $\quad$ male $=0$, female $=1$ ) and age exhibit statistically significant negative pairwise correlation with morning, afternoon, and evening stress, morning hunger, and feeling of being rested in the morning. Also, number of persons living in the participant's household is significantly negatively correlated with education level, morning hunger, hours of sleep, and morning, afternoon, and evening stress.

Our variable of interest, whether a participant overeats on a given day, is significantly correlated only with whether the participant overate the previous day. Surprisingly, none of the other single variables under consideration has a statistically significant correlation with overeating. This fact points to the need for multivariate analysis to investigate associations between groups of variables or stimuli and overeating, rather than individual variables alone.

We performed predictive modeling to determine overeating patterns and stimuli valid across pilot study participants. For this, we employed decision trees (described in Section 4.3). Figure 8 illustrates a sample decision tree obtained in our experiments. It states that females and unmarried males who did not overeat the previous day are not likely to overeat; married men who did not overeat the previous day are likely to overeat if they have had 7 or fewer hours of sleep. Among participants who did overeat the previous day, those who are either employed parttime or are married are likely to overeat. This tree achieves a prediction accuracy of $68 \%$ using 10 -fold cross-validation. A pruned version of this tree finds that married participants who overate the previous day are likely to overeat. This simplified tree achieves a $71.3 \%$ prediction accuracy, a significant improvement over the $63.5 \%$ accuracy obtained by the simplistic model that always predicts no overeating, the majority value of the overeating variable in our dataset.

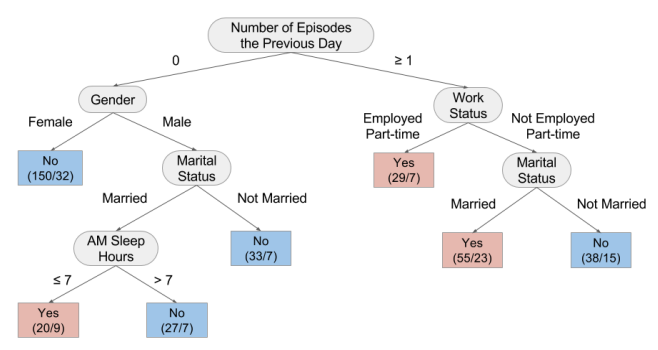

Figure 8. Sample Group-Based Decision Tree 
Table 2. Pearson Correlation Matrix

\begin{tabular}{|c|c|c|c|c|c|c|c|c|c|c|c|c|c|c|c|}
\hline & & $\begin{array}{c}\text { Gender 0: } \\
\text { male 1: } \\
\text { female }\end{array}$ & Age & Education & $\begin{array}{l}\text { Num. of } \\
\text { persons } \\
\text { living in } \\
\text { household }\end{array}$ & Income & $\begin{array}{c}\text { AM } \\
\text { Stress }\end{array}$ & $\begin{array}{l}\text { AM } \\
\text { Hunger }\end{array}$ & $\left|\begin{array}{c}\text { AM Feel } \\
\text { Rested }\end{array}\right|$ & $\begin{array}{c}\text { AM } \\
\text { Sleep } \\
\text { hours }\end{array}$ & $\begin{array}{c}\% \text { Weight } \\
\text { change } \\
\text { (from prev } \\
\text { day) }\end{array}$ & $\begin{array}{l}\text { PM } \\
\text { Stress }\end{array}$ & $\begin{array}{l}\text { EVE } \\
\text { Stress }\end{array}$ & $\begin{array}{c}\text { Num. of } \\
\text { Episodes } \\
\text { Previous } \\
\text { Day }\end{array}$ & $\begin{array}{c}\text { Did } \\
\text { overeat } \\
\text { today? }\end{array}$ \\
\hline \multirow{3}{*}{\begin{tabular}{|l|} 
Gender \\
$0:$ male \\
1:female
\end{tabular}} & Corr & 1 & $.325^{* *}$ & $-.136^{*}$ & $.205^{* *}$ & .063 & $-.211^{* *}$ & $-.230^{*+1}$ & -.176 & $-.123^{*}$ & .023 & $-.124^{*}$ & $-.203^{* \prime}$ & -.104 & -.089 \\
\hline & Sig. & & .000 & .010 & .000 & .238 & .000 & .000 & .001 & .023 & .665 & .024 & .000 & .052 & .096 \\
\hline & $\mathrm{N}$ & 352 & 352 & 352 & 352 & 352 & 343 & 343 & 343 & 343 & 342 & 331 & 295 & 352 & 347 \\
\hline \multirow[t]{3}{*}{ Age } & Corr & $.325^{4}$ & 1 & $-.539^{-1}$ & $.517^{* *}$ & $-.205^{* *}$ & $-.435^{* *}$ & $-.218^{*}$ & -.075 & -.057 & .007 & $-.318^{* \prime}$ & $-.329^{-*}$ & .059 & .058 \\
\hline & Sig. & .000 & & .000 & .000 & .000 & .000 & .000 & .167 & .289 & .896 & .000 & .000 & .273 & .279 \\
\hline & $\mathrm{N}$ & 352 & 352 & 352 & 352 & 352 & 343 & 343 & 343 & 343 & 342 & 331 & 295 & 352 & 347 \\
\hline \multirow[t]{3}{*}{ Education } & Corr & $-.136^{*}$ & $-.539^{* 1+}$ & 1 & $-281^{* \prime}$ & $.335^{*}$ & $.257^{*}$ & .312 & -.100 & $\begin{array}{l}.079 \\
\end{array}$ & .024 & $.203^{* *}$ & $.232^{*+}$ & $\begin{array}{ll}-.035 \\
\end{array}$ & $\begin{array}{l}.005 \\
\end{array}$ \\
\hline & Sig. & .010 & .000 & & .000 & .000 & .000 & .000 & .064 & .143 & .659 & .000 & .000 & .517 & .919 \\
\hline & $\mathrm{N}$ & 352 & 352 & 352 & 352 & 352 & 343 & 343 & 343 & 343 & 342 & 331 & 295 & 352 & 347 \\
\hline \multirow{3}{*}{\begin{tabular}{|l|} 
Num. of \\
persons \\
living in \\
household \\
\end{tabular}} & Corr & $.205^{-4}$ & $.517^{*+}$ & $-.281^{* *}$ & 1 & .057 & $-.273^{\text {t* }}$ & $-.394^{4}$ & -.055 & $-.213^{* \prime}$ & .025 & $-.181^{*}$ & $-.115^{*}$ & -.029 & .007 \\
\hline & Sig. & .000 & .000 & .000 & & .290 & .000 & .000 & .312 & .000 & .641 & .001 & .049 & .586 & .897 \\
\hline & $\mathrm{N}$ & 352 & 352 & 352 & 352 & 352 & 343 & 343 & 343 & 343 & 342 & 331 & 295 & 352 & 347 \\
\hline \multirow[t]{3}{*}{ Income } & Corr & .063 & -.205 & $.335^{*}$ & .057 & 1 & -.013 & .074 & $.175^{*}$ & -.037 & .045 & -.007 & .070 & .083 & .082 \\
\hline & Sig. & .238 & .000 & .000 & .290 & & .805 & .172 & .001 & .499 & .410 & .905 & .228 & .122 & .127 \\
\hline & $\mathrm{N}$ & 352 & 352 & 352 & 352 & 352 & 343 & 343 & 343 & 343 & 342 & 331 & 295 & 352 & 347 \\
\hline \multirow[t]{3}{*}{ AM Stress } & Corr & $-.211^{* t}$ & $-.435^{* *}$ & $.257^{* 1}$ & $-.273^{-1 *}$ & -.013 & 1 & $.159^{*+}$ & -.087 & .043 & .039 & $.696^{* t}$ & $.531^{* *}$ & -.090 & -.024 \\
\hline & Sig. & .000 & .000 & .000 & .000 & .805 & & .003 & .107 & .425 & .471 & .000 & .000 & .096 & .658 \\
\hline & N & 343 & 343 & 343 & 343 & 343 & 343 & 343 & 343 & 343 & 342 & 328 & 293 & 343 & 343 \\
\hline \multirow[t]{3}{*}{ AM Hunger } & Corr & $-.230^{-1}$ & $-.218^{* *}$ & $.312^{* *}$ & $-.394^{\prime \prime}$ & .074 & $.159^{* \prime}$ & 1 & $.107^{*}$ & $.191^{* *}$ & -.002 & .046 & .009 & -.006 & -.070 \\
\hline & Sig. & .000 & .000 & .000 & .000 & .172 & .003 & & .048 & .000 & .963 & .408 & .872 & .910 & 195 \\
\hline & $\mathrm{N}$ & 343 & 343 & 343 & 343 & 343 & 343 & 343 & 343 & 343 & 342 & 328 & 293 & 343 & 343 \\
\hline \multirow{3}{*}{\begin{tabular}{|l|} 
AM Feel \\
Rested
\end{tabular}} & Corr & $-176^{*}$ & -.075 & -.100 & -.055 & $.175^{* \prime}$ & -.087 & $.107^{*}$ & 1 & $.387^{* \prime}$ & .068 & -.097 & -.037 & .084 & .056 \\
\hline & Sig. & .001 & .167 & .064 & .312 & .001 & 107 & .048 & & .000 & .211 & .079 & .530 & .120 & .298 \\
\hline & $\mathrm{N}$ & 343 & 343 & 343 & 343 & 343 & 343 & 343 & 343 & 343 & 342 & 328 & 293 & 343 & 343 \\
\hline \multirow{3}{*}{\begin{tabular}{|l|} 
AM Sleep \\
hours
\end{tabular}} & Corr & $-.123^{\circ}$ & $\begin{array}{c}.057 \\
\end{array}$ & -.079 & -.213 & -.037 & .043 & .191" & .387 & 1 & $.129^{\circ}$ & -.008 & .006 & $-.110^{\circ}$ & -.027 \\
\hline & Sig. & .023 & .289 & .143 & .000 & .499 & .425 & .000 & .000 & & .017 & .888 & .917 & .042 & .612 \\
\hline & $\mathrm{N}$ & 343 & 343 & 343 & 343 & 343 & 343 & 343 & 343 & 343 & 342 & 328 & 293 & 343 & 343 \\
\hline \multirow{3}{*}{$\begin{array}{l}\% \text { Weight } \\
\text { change } \\
\text { (from prev } \\
\text { day) } \\
\end{array}$} & Corr & .023 & .007 & .024 & .025 & .045 & .039 & $\begin{array}{l}-.002 \\
\end{array}$ & $\begin{array}{l}.068 \\
\end{array}$ & $.129^{*}$ & 1 & -.031 & -.056 & -.047 & .014 \\
\hline & Sig. & .665 & .896 & .659 & .641 & .410 & .471 & .963 & .211 & .017 & & .577 & .341 & .390 & .803 \\
\hline & $\mathrm{N}$ & 342 & 342 & 342 & 342 & 342 & 342 & 342 & 342 & 342 & 342 & 327 & 293 & 342 & 342 \\
\hline \multirow[t]{3}{*}{ PM Stress } & Corr & $-.124^{*}$ & -.318 & $.203^{\prime \prime \prime}$ & $-181^{\prime \prime}$ & -.007 & $.696^{* t}$ & $\begin{array}{l}.046 \\
\end{array}$ & \begin{tabular}{|c|}
-.097 \\
\end{tabular} & -.008 & -.031 & 1 & $.624^{* *}$ & $\begin{array}{l}.039 \\
\end{array}$ & $\begin{array}{l}.084 \\
\end{array}$ \\
\hline & Sig. & .024 & .000 & .000 & .001 & .905 & .000 & .408 & .079 & .888 & .577 & & .000 & .481 & .129 \\
\hline & $\mathrm{N}$ & 331 & 331 & 331 & 331 & 331 & 328 & 328 & 328 & 328 & 327 & 331 & 291 & 331 & 331 \\
\hline \multirow[t]{3}{*}{ EVE Stress } & Corr & $-.203^{*+}$ & $-.329^{* *}$ & $.232^{* \prime}$ & $-.115^{*}$ & .070 & $.531^{* *}$ & .009 & \begin{tabular}{|c|}
-.037 \\
\end{tabular} & .006 & -.056 & .624 & 1 & -.029 & -.025 \\
\hline & Sig. & .000 & .000 & .000 & .049 & 228 & .000 & .872 & .530 & .917 & .341 & .000 & & .620 & .672 \\
\hline & $\mathrm{N}$ & 295 & 295 & 295 & 295 & 295 & 293 & 293 & 293 & 293 & 293 & 291 & 295 & 295 & 295 \\
\hline \multirow{3}{*}{\begin{tabular}{|l|} 
Number of \\
Episodes \\
Previous \\
Day \\
\end{tabular}} & Corr & $\begin{array}{l}-104 \\
\end{array}$ & $\begin{array}{l}.059 \\
\end{array}$ & -.035 & -.029 & .083 & -.090 & \begin{tabular}{|c|}
-.006 \\
\end{tabular} & .084 & $-.110^{*}$ & -.047 & -.039 & -.029 & 1 & $.330^{*+}$ \\
\hline & Sig. & .052 & .273 & .517 & .586 & .122 & .096 & .910 & .120 & .042 & .390 & .481 & .620 & & .000 \\
\hline & $\mathrm{N}$ & 352 & 352 & 352 & 352 & 352 & 343 & 343 & 343 & 343 & 342 & 331 & 295 & 352 & 347 \\
\hline \multirow{3}{*}{\begin{tabular}{|l} 
Did overeat \\
today?
\end{tabular}} & Corr & $\begin{array}{l}.089 \\
\end{array}$ & .058 & $\begin{array}{l}.005 \\
\end{array}$ & .007 & .082 & -.024 & $\begin{array}{l}.070 \\
\end{array}$ & .056 & $\begin{array}{l}-.027 \\
\end{array}$ & .014 & -.084 & $\begin{array}{l}.025 \\
\end{array}$ & $.330^{\circ}$ & 1 \\
\hline & Sig. & .096 & .279 & .919 & .897 & .127 & .658 & .195 & .298 & .612 & .803 & .129 & .672 & .000 & \\
\hline & $\mathrm{N}$ & 347 & 347 & 347 & 347 & 347 & 343 & 343 & 343 & 343 & 342 & 331 & 295 & 347 & 347 \\
\hline
\end{tabular}

${ }^{* *}$ : Correlation is significant (Sig.) at the 0.01 level (2-tailed), highlighted in dark green (+), dark blue (-).

*: Correlation is significant (Sig.) at the 0.05 level (2-tailed), highlighted in light green (-), light blue (-).

\subsection{Individual-based data analysis and personalized predictive modeling}

In addition to creating group-based predictive models, we generated a personalized decision tree predictive model for each participant that can identify stimuli that make the participant likely to overeat. We then used this personalized decision tree model to generate behavioral interventions to alert the participant about the potential for overeating when the app detects the presence of these stimuli. These interventions were delivered to the participants during the Feedback Phase. As an illustration, we describe here personalized predictive models for two sample participants, denoted as participants A and B.

Participant A is a single female in her 30's with some college education. She lost 10 pounds while 
using the app during the Assessment Phase. From her app-recorded data, we identified a significant negative correlation between occurrence of overeating episodes and number of sleep hours (Pearson Correlation $=-0.675$, with 2-tailed significance $=0.001)$. The decision tree constructed over her app-recorded data has a high prediction accuracy $(86 \%)$ and is very succinct, as depicted in Figure 9. On days that she reported having slept over 6 hours, she did not overeat (100\% accuracy). If she reported fewer than 6 hours of sleep, she was likely to overeat if she had no overeating episode the previous day. During her interview at the end of the Assessment Phase, she expressed full confidence in her ability to recognize what qualified as overeating: taking second servings, eating when already full, and having unhealthy or large snacks.

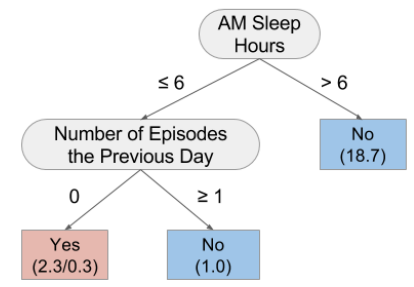

\section{Figure 9. Participant A's Decision Tree}

Participant B is a divorced male in his 40's with an Associate's degree. He experienced a net weight loss of 11 pounds while using the app during the Assessment Phase. During his interview at the end of this phase, he stated that he recorded restaurant meals and snacking on bread as instances of overeating. He expressed that he was aware of his overeating, but ate anyway. Nevertheless, he still managed to lose weight and reported greater awareness of overeating as a result of using the app. As depicted in the decision tree constructed over his app-recorded data in Figure 10, if he had weighed less than 212 pounds in the morning, had fewer than 8 hours of sleep, and was not stressed, he would not overeat.

Following this process, we created a personalized decision tree for each individual participant and corresponding intervention messages. Additional sample interventions are shown in Section 3.

\section{Discussion}

SlipBuddy was developed to help users identify and avoid stimuli that trigger overeating. During the Assessment Phase of the pilot study, participants consistently used the app and some continued using it after this phase ended even though they were no longer required to use it. This high utilization may be due to low burden associated with SlipBuddy use.

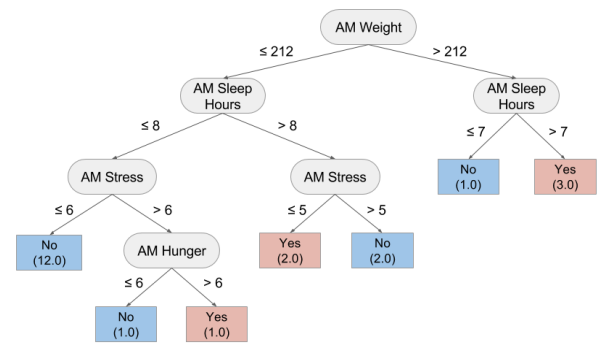

Figure 10. Participant B's Decision Tree

Most participants reported losing weight during the Assessment Phase even though weight loss was not intended or expected since the interventions were not provided during this phase. Weight loss during the Assessment Phase may indicate that SlipBuddy can be effective in making participants more aware of their own overeating and associated stimuli. This new awareness alone may result in behavior change. During the interviews conducted at the end of this phase, participants reported that the app made them more aware of their stress level, and some reported making healthier food/activity choices as a result.

Although most participants mentioned that they could easily identify when they were overeating, some participants reported difficulties determining what constituted an overeating episode (e.g., some recorded any snack outside of a meal, while others only recorded overeating episodes they considered "stress-eating"), which may account for some of the differences in number of episodes reported. Although we provide a clear definition of when a participant is supposed to press the Oops! button in the app (in Section 3.1), we learned that participants may benefit from further instruction and examples of what constitutes overeating.

Some participants reported that the three fixed check-in times did not correspond with their schedules, so they ended up completing multiple check-ins late in the evening. This presents a concern regarding the reliability of the self-reported stress levels, since participants are more likely to forget or misjudge their stress levels after some time has elapsed. Some participants reported weighing themselves only weekly or a few times throughout the course of the study, even though the app asked participants to enter weight daily. Therefore, we only considered weight loss based on the initial weight reported and the final weight reported at the end of the Assessment Phase. Although weekly weight fluctuations may provide interesting insights, our data was not reliable enough to consider this as a factor. We also collected location information associated 
with overeating episodes in the Assessment Phase. The participants had the option to turn off this feature. Our initial analysis of location data showed that although some participants provided location information, data cleaning needs to be performed on this data to identify meaningful insights.

\section{Conclusions and Future Work}

Slipbuddy app presents a novel approach to support weight loss; however it can be improved in a number of ways. Additional features that visually represent a user's progress over time can be added to the app. For example, the app could display a user's weight, number of overeating episodes, or stress over time. The app could provide graphs that are specific to interventions, allow users to quantify the intensity of overeating episodes using a scale or allow users to set goals for reducing overeating episodes gradually.

When it comes to stimulus control, the advantage of SlipBuddy over traditional counseling is that the app flags stimuli in the moment for the user and provides just-in-time intervention. In traditional counseling, patients are taught in sessions to identify stimuli that may be triggering unhealthy behaviors but they then must apply these skills independently in between sessions. The SlipBuddy app advances both technology and research paradigms by leveraging evidence-based behavioral strategies for weight loss, using passive sensing to detect contextual factors, minimizing user burden, and providing in-themoment intervention. This paper reports on the analysis of the data collected during the Assessment Phase only. The results of the Feedback Phase will help us better understand how stimuli control interventions can be delivered through SlipBuddy.

\section{Acknowledgements}

This research was supported in part by the National Science Foundation (NSF) under Grant CNS-1560229 REU SITE: "Data Science Research for Safe, Sustainable and Healthy Communities", and by the University of Massachusetts Science and Technology Initiatives Fund. Any opinions, findings, conclusions, and/or recommendations expressed in this paper are those of the authors and do not necessarily reflect the views of the NSF.

\section{References}

[1] World Health Organization. (June 15). Global Health Observatory (GHO) Data Available: http://www.who.int/gho/ncd/risk_factors/obesity_text/en/

[2] K. M. Flegal, D. Kruszon-Moran, M. D. Carroll, C. D. Fryar, and C. L. Ogden, "Trends in obesity among adults in the united states, 2005 to 2014 ," JAMA, vol. 315 , pp. 2284-2291, 2016.

[3] Diabetes Prevention Program Research Group, "Reduction in the Incedence of Type 2 Diabetes with Lifestyle Intervention or Metformin," The New England journal of medicine, vol. 346, pp. 393-403, 2002.

[4] The Diabetes Prevention Program (DPP) Research Group, "The Diabetes Prevention Program (DPP): Description of lifestyle intervention," Diabetes care, vol. 25, pp. 2165-2171, 2002.

[5] C. G. Fairburn and E. R. Rothwell, "Apps and eating disorders: A systematic clinical appraisal," The International Journal of Eating Disorders, vol. 48, pp. 1038-1046, 02/27

01/30/accepted 2015.

[6] C. Free, G. Phillips, L. Galli, L. Watson, L. Felix, P. Edwards, et al., "The effectiveness of mobile-health technology-based health behaviour change or disease management interventions for health care consumers: a systematic review," PLoS med, vol. 10, p. e1001362, 2013.

[7] S. Pagoto, K. Schneider, M. Jojic, M. DeBiasse, and D. Mann, "Evidence-based strategies in weight-loss mobile apps," American journal of preventive medicine, vol. 45, pp. 576-582, 2013.

[8] B. Y. Laing, C. M. Mangione, C.-H. Tseng, M. Leng, E. Vaisberg, M. Mahida, et al., "Effectiveness of a smartphone application for weight loss compared to usual care in overweight primary care patients: a randomized controlled trial," Annals of internal medicine, vol. 161, pp. S5-12, 2014.

[9] A. S. Juarascio, S. P. Goldstein, S. M. Manasse, E. M. Forman, and M. L. Butryn, "Perceptions of the feasibility and acceptability of a smartphone application for the treatment of binge eating disorders: Qualitative feedback from a user population and clinicians," International journal of medical informatics, vol. 84, pp. 808-816, 06/15 2015.

[10] A. S. Juarascio, S. M. Manasse, S. P. Goldstein, E. M. Forman, and M. L. Butryn, "Review of Smartphone Applications for the Treatment of Eating Disorders," European eating disorders review : the journal of the Eating Disorders Association, vol. 23, pp. 1-11, 10/10 2015.

[11] L. E. Burke, J. Wang, and M. A. Sevick, "Selfmonitoring in weight loss: a systematic review of the literature," Journal of the American Dietetic Association, vol. 111, pp. 92-102, 2011.

[12] K. J. Saunders and D. C. Williams, "StimulusControl Procedures," in Handbook of Research Methods in Human Operant Behavior, K. A. Lattal and M. Perone, Eds., ed Boston, MA: Springer US, 1998, pp. 193-228.

[13] M. Hall, E. Frank, G. Holmes, B. Pfahringer, P. Reutemann, and I. H. Witten, "The WEKA data mining software: an update," $A C M$ SIGKDD explorations newsletter, vol. 11, pp. 10-18, 2009 